\title{
Carnets
}

Revue électronique d'études françaises de l'APEF

Deuxième série - 7| 2016

Plurilinguisme et migrations dans la littérature de langue française

\section{La météorologie du sensible chez Nina Bouraoui}

\section{Loucif Badreddine}

\section{(2) OpenEdition}

Journals

\section{Édition électronique}

URL : http://journals.openedition.org/carnets/928

DOI : $10.4000 /$ carnets. 928

ISSN : 1646-7698

Éditeur

APEF

Référence électronique

Loucif Badreddine, «La météorologie du sensible chez Nina Bouraoui », Carnets [En ligne], Deuxième série - 7 | 2016, mis en ligne le 31 mai 2016, consulté le 20 avril 2019. URL : http://

journals.openedition.org/carnets/928; DOI : 10.4000/carnets.928

Ce document a été généré automatiquement le 20 avril 2019.

\section{(c) (i) \&)}

Carnets est mis à disposition selon les termes de la licence Creative Commons - Atribution - Pas d'utilisation commerciale 4.0 International. 


\title{
La météorologie du sensible chez Nina Bouraoui
}

\author{
Loucif Badreddine
}

\author{
Quand je suis seul, je ne suis pas seul, mais, dans ce \\ présent, je reviens déjà à moi sous la forme de \\ Quelqu'un. Quelqu'un est là, où je suis seul.
}

(Blanchot, 1955 : 22)

1 Le $\mathrm{xx}^{\mathrm{e}}$ siècle littéraire s'est caractérisé par l'éclatement des genres. Le roman, l'autobiographie comme la poésie ont perdu de leurs rigidités intangibles pour offrir, dans de nouvelles combinaisons, une régénérescence féconde. Pour illustrer ce constat, nous allons nous intéresser au cas de Poupée Bella ${ }^{1}$, le journal intime de Nina Bouraoui.

2 Comme genre, c'est le signe de la modernité et l'évolution de notre société qui devient de plus en plus individualiste. Naissant vers la fin du XIII ${ }^{\mathrm{e}}$ siècle, il ne prend son véritable essor, vers le $\mathrm{xIX}^{\mathrm{e}}$ siècle, qu'avec l'émancipation de l'individu, bourgeois, vers sa singularisation et son indépendance du religieux et du spirituel au temps où « la société moderne tente de canaliser les pulsions et les émotions par la culture du contrôle de soi » (Marie Bonnet, 2007). Le journal intime a l'ambition de toucher l'universel par le personnel, l'ultime par l'intime.

3 Un journal intime, comme récit de soi, n'est pas destiné a priori, dans son principe même de secret caché, à une éventuelle vulgarisation. L'authentique journal intime ne se donne à lire qu'individuellement puisqu'il n'en existe qu'une seule copie manuscrite, celle qu'une personne a rédigée dans la solitude de la nuit, dans l'intimité de son lit où elle raconte tout ce qui a échappé au visible, à la sphère du social et qui relève du privé, de l'identitaire et de l'existentiel.

4 Nina Bouraoui, avec Poupée Bella, est sur les traces de Julien Green², Pourquoi suis-je moi? (Green, 1996), ou celle d'Henri-Frédéric Amiel qui écrivit «C'est une existence déracinée et disloquée que la mienne et je ne sais vraiment plus pourquoi je vis » (Amiel, 1986: 660). Mais la comparaison avec ses célèbres et volumineux journaux intimes, tenus au jour le jour, s'arrête là. Dans le cas précis de notre corpus, il s'agit formellement d'un journal intime et substantiellement d'un récit autobiographique. Un truchement de genres qui 
reflète les différentes identités (culturelle, sexuelle, nationale et surtout littéraire) profondément indécises de la diariste qui relève de sa difficulté à construire à travers ce texte une quelconque téléologie romanesque, puisqu'elle n'a pas choisi le genre autobiographique explicitement. Nous allons de même essayer de dévoiler la dimension clairement poétique située sur le plan scriptural qui donne à ce texte une affectation mélodique qui appuie fortement son épaisseur mélancolique.

Nombreux sont les travaux sur le journal intime. On en retiendra l'étude de Michèle Leleu ${ }^{3}$ des différents caractères des diaristes en distinguant le sentimental du nerveux, du passionné etc., ou celle de Béatrice Didier (Didier, 1976), plus approfondie à notre sens, qui a entrepris son étude du point de vue sociocritique, psychanalytique et structural. Notre travail s'appuiera sur les différentes définitions ${ }^{4}$ proposées par B. Didier tout en nous inspirant de la conception philosophique de Blanchot du journal intime et s'inscrira dans une perspective rhématique pour identifier les traits formels qui caractérisent cette forme de « récit de soi" comme littérature personnelle, mais aussi axiologique pour en déterminer la valeur poétique.

Le journal intime, comme l'affirmait Blanchot, est un refus d'un auteur de se « dessaisir de lui-même » (Blanchot, 1955 : 19). Le même auteur peut le faire plus volontairement lorsqu'il s'agit d'un autre genre, le roman par exemple, plus impersonnel que le journal intime. Il s'agit donc d'une distance entre l'auteur et lui-même qui va déterminer son écriture : de l'intime jusqu'à l'impersonnel, exposé avec un certain recul. Chez Bouraoui, cette distance est très réduite. La diariste n'a pas pu se dessaisir complètement d'ellemême, elle a pris le dessus sur son texte, devenu malgré lui fragmentaire et ambigu.

Dans Poupée Bella, les faits - s'ils peuvent être désignés ainsi - se déroulent à Paris, presque exclusivement dans des clubs homosexuels. Les deux derniers mois de l'année 1987, toute l'année de 1988, jusqu'à la journée du 21 juin 1989, sont racontés dans la solitude de la nuit ${ }^{5}$ seize ans plus tard par Nina Bouraoui, d'où le premier truchement du genre avec l'autobiographie. Bien que tous deux appartiennent à la même souche du "récit de soi", ils n'en demeurent pas moins différents: le premier s'inscrit dans l'immédiateté, le deuxième dans la rétrospection. Pour arriver à une véritable écriture de soi désignée génériquement par «journal intime », il faut qu'il y ait une immédiateté entre l'instant vécu et l'instant de l'écriture de ce même vécu. Une fois ce moment passé, le soi a changé. Ce «nouveau » soi raconte un "ancien » soi qu'il regarde différemment comme s'il s'agissait d'un Autre. Le diariste, qui ne l'est plus techniquement, raconte, dès lors, des souvenirs différés, et non son vécu immédiat. Cette écriture n'est plus sur le vif, charnelle, physique et émotionnelle, mais intellectualisée par la mémoire qui restitue le passé, ou ce qu'elle en a gardé, à partir d'un autre soi, du présent, au moment de le restituer. Les recherches ${ }^{6}$ en neurosciences le confirme : la mémoire nous joue des tours : les trous de mémoire, la mémoire sélective, les mécanismes de défense psychologique etc. Toutes ces variables combinées les unes avec les autres participent à ce changement de soi.

8 C'est donc ce décalage entre les deux temps, celui du vécu et celui de l'écriture, qui fait de Poupée Bella un journal intime feint. Nous nous permettons le choix de «feint ", plutôt que celui de "faux journal intime», car nous estimons que la sincérité est effective dans la mesure où ce journal ne possède pas de vision totalisante, qui traduit un manque de recul, empêchée par l'immédiateté d'une écriture différée qui n'a rendu compte de la réalité que fragmentairement. D'ailleurs, le déploiement fictionnel - qui reste d'ailleurs minimaliste mais suffisant pour l'apparenter à l'autobiographie, le seul habilité à autoriser cette 
totalisation - est gêné par les brisures thématiques et matérielles. Elle a gardé ses souvenirs en bribes, sans jointures narratives. Le résultat a été donc une restitution obligée par l'immédiateté feinte et une construction facilitée par le différé et l'ajournement qui permet plus de réflexion mature, qui débarrasse les sentiments de la confusion du moment confronté à l'oubli de ce que le cerveau estime être du superflu. Bouraoui a voulu figer, grâce à la fragmentation, des images et des sensations, telles des instantanés, pour les isoler et les mettre en évidence. Sans cela, ces images seraient moins nettes, diluées dans le flux d'une écriture continue. Elle dénonce à sa manière « l'illusion biographique, qui consiste à croire qu'une vie vécue peut ressembler à une vie racontée » (Sartre, $1995: 279$ ).

9 Le rapprochement entre ce qui est raconté et son référent réel (le vécu) n'est pas du même ordre que celui qui existe chez les autres diaristes ${ }^{7}$; dans Poupée Bella, il est compromis. Voilà ce qu'on peut lire à l'entrée du 23 janvier 1988 :

Je cherche. Je cherche la femme de ma vie dans la nuit. Je cherche, dans la forêt. Je cherche, sous les vagues. Je cherche après les dunes. J'ai un destin amoureux. J'ai plusieurs vies. J'ai plusieurs corps sous mon corps. Je suis au début de tout. Je suis à la fin des jours heureux. (Bouraoui, $2004: 21$ )

10 On voit bien à travers cet extrait, non loin d'être exceptionnel, que la mise en mots de la réalité est fortement imagée. La diariste n'a pas l'intention de raconter sa biographie réelle, mais d'en proposer une version poétisée. Plusieurs indices appuient ce choix à commencer par la forme fragmentaire, courte, une syntaxe rythmée et surtout des répétitions lexicales et sonores réalisées par des allitérations : «Dans ma tête les tours de Créteil comme des puits de solitude. La nuit des filles est infinie. La nuit de Julien est une nuit fermée. Je pourrais écrire à Julien. Je pourrais l'insulter. » (Bouraoui, 2004 : 113).

11 L'anaphore, très utilisée, presque systématiquement, donne du rythme aux phrases, met l'accent sur les mots telle une obsession, et suggère une palette d'impressions allant du plaidoyer à la légèreté de la litanie, du mélancolique à l'euphorique : «J'attends l'amour dans mes mains. J'attends l'amour dans mon corps entier. J'attends une fille que je ne connais pas. J'ai besoin d'un corps contre le mien. J'ai besoin d'une chair qui répondrait à la mienne.» (Bouraoui, 2004: 25) Mais c'est sous forme d'expolition qu'elle essaye de rendre compte d'une seule angoisse, » un désir d'être qui ne pourra s'accomplir, (...) que dans l'écriture et l'amour ${ }^{8} "$ (Bouraoui, 2004 : quatrième de couverture). Elle réitère ce désir comme une essence sous toutes ses formes de sublimation les plus diverses: le corps, l'homosexualité, soi et les autres, le tout par rapport à son écriture.

12 Cette dimension poétique, obligatoirement subjective, montre bien que c'est résolument une écriture de soi qui a imposé, comme une évidence, le choix du journal intime, comme seule forme pouvant contenir cette écriture indispensablement fragmentaire, exigée par un discours sur un soi multiple. Une fragmentation « liée à la mobilité de la recherche, à la pensée voyageuse qui s'accomplit par affirmations séparées et exigeant la séparation " (Blanchot, 1961 : 186). Les allers-retours de sa jeunesse et de sa préadolescence entre deux lieux ${ }^{9}$, deux cultures, deux religions différentes, ont empreint son identité de disparités et de contradictions même. Ici on exprime, là-bas on tait; l'une exhibe, l'autre dissimule. Elle, une fille timide, cherchait seulement à être anonyme, n'attirant pas l'attention, dans un univers majoritairement masculin ${ }^{10}$ et brutal. Une vie pleine de contrainte et d'opposition entre deux cultures incarnées par une mère occidentale et un père oriental. Elle s'est alors réfugiée dans une écriture où le «je» n'est autre que l'auteure-narratrice-personnage compris comme un monème tri-syntagmatique figé. Une 
sorte de détachement de soi pour se voir dans un premier temps, et dans un deuxième pour s'écrire: une exposition de soi à soi-même, aperçu par un troisième "soi ». Un doublement triple qui caractérise souvent les diaristes. Qui est-elle ? Celle qui écrit, celle qui vit, ou celle décrite (écrite) dans son journal intime? Ou les trois à la fois?

«Maîtriser son écriture c'est se maîtriser soi » (Bouraoui, 2004 : 83), «Écrire est aussi une façon de se rassembler, de se retrouver à l'intérieur de soi » (Bouraoui, 2004 : 112). Dès la première page du journal, elle se découvre inconsciemment: «je danse seule devant le miroir de la chambre, je n'ai rien de silencieux en moi, tout bouge, tout crie » (Bouraoui, 2004 : 7). Lorsqu'on est dans un récit de soi, cette image du miroir est plus parlante que dans un autre genre de récit. Cela évoque "le stade du miroir ", une étape importante dans la formation psychologique de l'homme. Ce stade lui permettra d'avoir conscience de son unité d'une part, et, de l'autre, il est à comprendre « comme une identification au sens plein (...) à savoir la transformation produite chez le sujet, quand il assume une image » (Lacan, 1949: 50). Dolto a étendu le concept du miroir à tout ce qui se reflète à partir de ce que dégage le moi ; cela peut être un commentaire, un regard, etc., nécessaire à la construction de soi, à l'image totalisante, où Bouraoui se rassemblerait grâce à l'écriture, justement.

Elle écrit donc. «J'ai perdu la foi. Je retrouve l'écriture. J'ai perdu le désir. Je retrouve le journal intime » (Bouraoui, 2004 : 117). « L'écriture prend dans le Milieu des Filles. C'est la seule façon, pour moi, de devenir une personne" (Bouraoui, 2004: 42), "[o]n devrait pouvoir tout écrire, d'une écriture qui viendrait de l'intérieur de soi, une écriture secrète et inédite " (Bouraoui, 2004: 141). "Je démonte un système: je déteste ce que j'écris, je déteste ce que je suis » (Bouraoui, $2004: 171)$. On pourrait former une véritable anthologie qui se rapporterait au thème de l'écriture dans Poupée Bella qui laissera croire - d'après l'affirmation suivante : "Je viens de l'écriture » (Bouraoui, 2004:139) - que son identité se conçoit en s'écrivant. «Je suis en devenir homosexuel comme je suis dans le livre en train de se faire » (Bouraoui, 2004 : 49). Par livre, on ne peut pas entendre que Poupée Bella , mais bien tout écrit, son œuvre, jusqu'à Standard ${ }^{11}$, nettement autobiographique.

15 Chez Bouraoui, l'écriture scelle la relation entre le raconté et le vécu ou se substitue catégoriquement à elle. Son identité personnelle, et plus particulièrement sexuelle (l'objet visé dans Poupée Bella), prend ses traits à travers son écriture et se façonne par les histoires qu'elle ne cesse de raconter. C'est ce que Paul Ricœur appelle identité narrative. Pour lui « le temps devient temps humain dans la mesure où il est articulé de manière narrative » (Ricoeur, 1983 : 17). L'identité humaine, en tant que constante qui caractérise une seule personne lui assurant son individualité et sa subjectivité, c'est-à-dire tout ce qui caractérise la permanence de cette identité (sa mêmeté, dira Ricoeur), ne peut être réalisée que grâce à la mémoire mise en mots qui doit s'accomplir avec, un tant soi peu, une dimension narrative suffisante pour construire un récit, une histoire sur soi, capable d'unifier tous les différents moments parcellaires en les subsumant sous les traits de l'identité personnelle.

16 Cet être, dans cette perspective, est un être-là, plus proche de la définition du Dasein $^{12}$, défini moins par son aspect substantiel que par son aspect relationnel, puisqu'elle est sans cesse dans l'interaction. C'est son image et son unité qui se façonne grâce à l'écriture, mais toujours en coopération avec autrui. En effet, la diariste, tout en dévoilant son intimité, se soumet au jugement de l'Autre. Et c'est pour cela peut-être qu'elle a choisi avec minutie, en favorisant ce genre, celui à qui elle se dévoile. Le journal intime impose, tel un pacte secret, la discrétion. Son discours est supposé être chuchoté dans l'intimité 
restreinte et exclusive d'une seule personne. Tous ceux qui veulent être dans la confidence viennent avec toute empathie, puisqu'ils le font non sans le remord d'un voyeur qui n'est pas autorisé à le faire. La publication de cette intimité est une forme de confession, de justification, une main tendue vers l'autre, une tentative pour interpeller sa sympathie, de s'inscrire dans l'extériorité socialisée qui dicte les normes pour l'implorer et s'expliquer pour des choix autres que ceux admis par la société. La diariste veut non seulement se dévoiler, mais s'exhiber à outrance, en donnant moult informations pour se rendre ordinaire, visible, trop visible pour se banaliser en vue des normes sociétales, une autre manière de se dessaisir de soi. «Toutes les filles veulent devenir célèbres, pour réparer l'homosexualité » (Bouraoui, $2004: 47)$. » Dans mon cas, il faut écrire pour se faire aimer " (Bouraoui, 2004 : 45). C'est une honte manifeste qu'elle éprouve : "Chaque fois, avant d'entrer dans une boîte de filles, j'ai peur; parce que j'ai rendez-vous avec ma conscience (...). Il faut un temps, pour s'adapter, pour accepter aussi. Il faut un temps pour oublier son éducation. Il faut du temps pour assumer son plaisir» (Bouraoui, 2004 : 51-52). L'Autre est constamment présent : «Il y a parfois une honte à être homosexuelle, à cause des autres, à cause de l'échec amoureux » (Bouraoui, 2004 : 37). «Il m'arrive de changer de prénom dans la nuit. Il m'arrive d'avoir honte de moi » (Bouraoui, 2004 : 49). D'ailleurs, elle a attendu jusqu'à son arrivée à Paris afin de trouver le courage pour franchir le pas, et de déclarer enfin « [j]e suis dans le temps du Milieu des Filles. Je suis dans le temps de mon homosexualité » (Bouraoui, 2004:17). Elle a besoin de la complicité des autres pour se sentir dans son milieu ; comme l'affirmait, pour d'autres raisons, Sartre : «j'ai besoin d'autrui pour saisir à plein toutes les structures de mon être " (Sartre, 1943 : 277). À Rennes ${ }^{13}$, on ne lui ressemble pas; le regard de l'autre est plus présent, plus pressant, à cause du niveau d'urbanisation qui empêche tout retrait et circonspection impersonnelle. La vie qu'elle souhaitait a commencé réellement lorsqu'elle a franchi la porte du Katmandou ${ }^{14}$, le monde de la nuit. Un monde qui lui ressemble, où elle aurait certes un peu honte mais ne culpabiliserait pas pour autant; la honte étant ce qui est en relation avec les valeurs socioculturelles (relative à son être), et la culpabilité ce qui est en relation avec un idéal de soi (relative à son advenir).

17 L'identité personnelle est à prendre, dans le cas précis de cette auteure qui raconte son homosexualité, comme une identité psychique et physiologique, non dans leur dualité matérialiste/mentaliste, mais dans leurs confusions qui vont engendrer cet être-là unique avec une sensibilité singulière. C'est l'histoire d'une tranche de vie, dans le monde des filles, racontée à travers un corps, citée fort régulièrement, plus de 170 occurrences. «C'est l'odeur d'abord, l'odeur des corps, (...) c'est la voix d'une femme, (...) ce sont les yeux, ce sont les mains, les petites marches à monter, c'est un spectacle, c'est le Milieu des Filles » (Bouraoui, $2004:$ ).

Sans exagération aucune, on pourrait dire en conséquence que c'est l'histoire d'un corps : «Je suis dans la seule vérité. La vérité de mon corps » (Bouraoui, $2004: 16$ ), «j'entre avant minuit au Katmandou, club féminin, j'entre sous la terre, j'entre dans mon corps » (Bouraoui, 2004: 8). «L'écriture est comme l'amour, elle passe par le corps »; « [j]'ai envie d'écrire comme je pourrais avoir envie d'un corps » (Bouraoui, 2004: 41). Un corps qui n'existe que dans le milieu des filles, un corps homosexuel capable de ressentir la chaleur, la sensualité, et peut-être même de se sublimer en un objet de spéculation sentimentale et charnelle : « je veux savoir combien je vaux, combien je peux espérer de ce corps-là » (Bouraoui, 2004: 9), ou tout simplement de désir : «je pourrais embrasser n’importe qui. Je veux juste une voix qui répétera mon nom » (Bouraoui, 2004: 9); «je 
suis la proie. Je suis l'appât » (Bouraoui, 2004: 10), «[j]e deviens un corps qui danse " (Bouraoui, 2004 : 11), « [j]e suis là. Regardez-moi » (Bouraoui, 2004 : 11). Ailleurs, le reste du temps, dans un autre territoire en dehors de ce milieu, l'auteure n'en mentionne aucune existence, comme effacée.

Bouraoui, par son corps, devient manifestation de son expression émotionnelle, une sorte de corporéité expressive et une territorialisation revendicative qui est passée par l'affirmation communautaire faisant de ce texte un témoignage, puisqu'elle raconte une brève période de sa vie qu'elle considère comme une expérience à partager avec d'autres lecteurs qui pourraient être dans une situation semblable. Poupée Bella est aussi un recueillement de soi ; cependant, loin d'être solipsiste, il révèle une intériorité, non pas dans sa confrontation avec l'extériorité, mais seulement pour marquer sa différence avec elle ; puisqu'au fond c'est la présence de l'Autre - et son intimité - qui définit la sienne.

Elle est tout cela à la fois, Nina Bouraoui. En permanence entre le désir intime d'un amour et le désir dévoilé d'une écriture, entre un être existant et un être en advenir. Son journal intime l'est aussi entre le brouillon (pas dans le sens reçu, mais au sens d'essai ou de note) et un récit de soi murement réfléchi. Entre la gêne du dévoilement de ce qui aurait dû rester secret, et la délivrance de ce même secret. Mais aussi l'inachevé publié, comme l'est ce journal intime; fragmentaire, tel que défini par Blanchot, où la circonscription moralisatrice est très élevée, fréquente, avec des pensées aphoristiques ambigües; " concentrée, obscurément violente, qui à titre de fragment est déjà complète (...) c'est étymologiquement l'horizon, un horizon qui borne et qui n'ouvre pas " (Blanchot, 1990 : 186), illustré par les passages suivants : «il n'y a aucun malheur homosexuel. Il n'y a qu'un malheur amoureux» (Bouraoui, 2004: 33); «la main qui écrit est une main qui guérit » (Bouraoui, $2004: 126$ ) ; « la nuit est une leçon » (Bouraoui, $2004: 127$ ); « il y a une vraie beauté dans un corps qui se cache » (Bouraoui, $2004: 127$ ) ; « [i]l n'y a que des corps désirables dans la nuit» (Bouraoui, 2004: 42); «[o]n ne se remet pas de son premier amour, comme on ne doit pas se remettre de son premier livre » (Bouraoui, 2004:40).

En définitive, chez Nina Bouraoui et grâce à l'analyse rétroactive et au travail introspectif qu'a permis son journal intime, la connaissance de soi est devenue une volonté consciente mise en acte par une écriture, non comme intermédiaire par lequel elle se chercherait, mais comme un appareil qui instaure une réflexion sur un soi qui se cherche. Analogue au cogito cartésien, son « $\mathrm{je}^{15}$ " permanent (dans la grande majorité de son œuvre et très particulièrement dans ses cinq premiers romans), omniprésent, a besoin d'écrire pour arriver à la conscience de soi. Pour elle, l'amour, le corps, c'est-à-dire le désir sous toutes ses formes, est de la même nature passionnelle et intimiste que l'écriture.

\section{BIBLIOGRAPHIE}

AMIEL, Henri Frédéric (1986). Journal Intime, Tome VI. Lausanne : L'Age d'homme.

BLANCHOT, Maurice (1955). L'Espace littéraire. Paris : Gallimard. 
BLANCHOT, Maurice (1990). « Mémorandum sur "le cours des choses" », texte préparatoire pour la Revue internationale [vers 1961], Lignes, $\mathrm{n}^{\circ} 11$, septembre, pp. 185-186.

BONNET, Marie (2007). « À la recherche de l'émotion perdue (revue Face à Face, 2006, 8-9) »

[Comptes rendus d'ouvrages] [en ligne], Ethnographiques.org. [consulté le 11.07.2015]

<URL : http://www.ethnographiques.org/2007/Bonnet>

Bouraoui, Nina (2004). Poupée Bella. Paris : Stock.

BOURAOUI, Nina (2014). Standard. Paris : Flammarion.

CASTELLO, Edna (2004). « L'écriture, une pratique amoureuse. » [en ligne]. Magazine 360. [consulté le 03.06.2015]

<URL : http://www.univers-l.com/interview_nina_bouraoui.html>

DIDIER, Béatrice (1972). Le Journal intime. Tunis : Cérès Édition.

HEIDEGGER, Martin (1964). Etre et temps. Paris : Gallimard.

LELEU, Michèle (1952). Les Journaux intimes. Paris : PUF.

LELEU, Michèle (1965). « Une “météorologie intime”, le Journal de Charles Du Bos », Cahiers de

l'Association internationale des études françaises, $n^{\circ}$ 17, pp. 133-150.

GREEN, Julien (1996). Pourquoi suis-je moi ? :Journal [XVI] 1993-1996. Paris : Fayard.

RIC@UR, Paul (1983). Temps et récit, tome I. Paris : Le Seuil.

SARTRE, Jean-Paul (1943). L'Etre et le Néant. Paris : Gallimard.

SARTRE, Jean-Paul (1995). Carnets de la drôle de guerre. Paris : Gallimard (nouvelle édition).

SIMONNET, Dominique (2004). «Écrire, c'est retrouver ses fantômes » [en ligne], L'Express magazine. [consulté le 03.06.2015]

<URL : http://www.lexpress.fr/culture/livre/ecrire-c-est-retrouver-ses-fantomes_819681.html>

TULVING, Endel (1995). « Organisation of memory: quo vadis? », The Cognitive Neurosciences, MIT

Press, pp. 839-847.

TULVING, Endel (2004). «La mémoire épisodique : de l'esprit au cerveau », Revue Neurol, $n^{\circ} 160$, pp. 9-23. (EUSTACHE F, DESGRANGES B, VIADER F, trad.).

\section{NOTES}

1. Bouraoui, Nina (2004). Poupée Bella. Paris : Stock. Toutes les références concernant cet ouvrage renverront à cette édition avec l'abréviation P.B.

2. Lui aussi a été marqué par son homosexualité, comme Bouraoui, comme nous allons le voir.

3. Au titre explicite : Les Journaux intimes. Leleu est aussi l'auteure d'un article qui a inspiré le titre du nôtre : Une « météorologie intime », le Journal de Charles Du Bos.

4. Celle du journal intime en plus des autres récits de soi, mais surtout la question du décalage ou la « simultanéité dans le journal entre le fait et l'écrit » (Didier, 1976:7) qui nous sera utile dans cet article.

5. De l'aveu même de Nina Bouraoui dans une interview donnée à Edna Castello. (Castello, 2004).

6. Citons ceux d'Endel Tulving qui concernent l'organisation de la mémoire (Tulving, 1972), ou celui de la mémoire épisodique (Tuliving, 2004) 
7. Pour ne citer que les plus célèbres d'entre eux, c'est-à-dire Amiel, Green ou Anne Frank, il y a une restitution radicale de la réalité. En plus des complaintes et des atermoiements successifs qui caractérisent la plupart des journaux intimes.

8. C'est nous qui soulignons.

9. L'Algérie et la France.

10. «Quand je faisais les courses avec mon père, je mettais un jogging, j'avais les cheveux très courts et une apparence androgyne, et j'étais très fière. Je me disais : "La vraie vie est de ce côtélà !" (Simonnet, 2004) affirmait Bouraoui dans une interview donnée à Dominique Simonnet.

11. Son dernier roman en date.

12. Tel que défini par Heidegger dans Être et Temps, notamment dans cette citation où «les structures de l'être-là [...] se confondent avec celles de l'être-au-monde : l'être-avec-autrui et la coexistence » (Heidegger : 1964 : 144).

13. La ville qui l'a vu grandir.

14. Un célèbre club homosexuel féminin à Paris.

15. Rien que pour la première entrée de Poupée Bella, il y a cinquante occurrences de "je». Rappelons que ce Journal fait 151 pages.

\section{RÉSUMÉS}

Née entre deux cultures, occidentale et orientale, Nina Bouraoui a écrit un journal intime qui lui ressemble. Poupée Bella possède les caractéristiques du journal intime, mais aussi ceux d'un récit autobiographique. Nous avons noté de même quelques traces qui le rattacheraient au témoignage. Ce qui est sûr, c'est son appartenance à la sphère du "récit de soi ", mais encore sa valeur poétique indéniable qui montre la sensibilité de l'auteure et son véritable amour pour l'écriture.

Born between two cultures, Western and Eastern, Nina Bouraoui wrote a diary that resembles her. Poupée Bella has the characteristics of a diary, but also those of an autobiographical narrative. We can also find in it some traces of a testimony. What is certain is it belongs to the sphere of "self-narrative", and it has an undeniable poetic value that shows the sensitivity of the author and her true love for writing.

\section{INDEX}

Keywords : Bouraoui (Nina), diary, self-narrative, Poupée Bella, identity

Mots-clés : Bouraoui (Nina), journal intime, récit de soi, Poupée Bella, identité

\section{AUTEUR}

\section{LOUCIF BADREDDINE}

Université de Khenchela

loucifbadre[at]gmail.com 